\title{
STATISTICAL CAUSALITY AND STABLE SUBSPACES OF $H^{P}$
}

\author{
LJILJANA PETROVIĆ and DRAGANA VALJAREVIĆ $\bowtie$
}

(Received 17 May 2012; accepted 23 May 2012; first published online 1 August 2012)

\begin{abstract}
In this paper we consider the statistical concept of causality in continuous time between filtered probability spaces, based on Granger's definitions of causality. Then we consider some stable subspaces of $H^{p}$ which contain right continuous modifications of martingales $P\left(A \mid \mathcal{G}_{t}\right)$. We give necessary and sufficient conditions, in terms of statistical causality, for these spaces to coincide with $H^{p}$. These results can be applied to extremal measures and regular weak solutions of stochastic differential equations.
\end{abstract}

2010 Mathematics subject classification: primary 60G44; secondary 60H10.

Keywords and phrases: filtrations, causality, stable subspaces, extremal measures, stochastic differential equations.

\section{Introduction}

In this paper we consider stable subspaces of $H^{p}$ which contain right continuous uniformly integrable $\left(\mathcal{F}_{t}, P\right)$-martingales, and we investigate their connection with the concept of causality.

In Section 2 we give some definitions and basic properties of the causality concept (see [14]) and we recall some general facts concerning stable subspaces of martingales (see [16]), which we will use later.

The given causality concept is shown to be equivalent to a generalisation of the notion of weak uniqueness for weak solutions of stochastic differential equations (see [13]). In [14] it is shown that the given causality concept is closely connected to the extremality of measures and the martingale problem. Also, the preservation of the martingale property, if the information $\sigma$-algebra increases, is shown to be strongly connected to the concept of causality (see [1]). Moreover, in [17], equivalence is proved between the given concept of causality and orthogonality of the local martingales.

Stable subspaces were investigated in [10], where it is proved that elements of stable $(G)$ (the smallest stable subspace of $H^{p}$ containing $G$ ) remain martingales under

The work is supported by the Serbian Ministry of Science and Technology (Grants 044006 and 179005).

(c) 2012 Australian Mathematical Publishing Association Inc. 0004-9727/2012 \$16.00 
any measure $Q$ which is absolutely continuous relative to $P$. Some results from [6], concerning stable subspaces of some topological spaces of martingales associated with Markov processes, are improved in [4]. Specifically, in [4] a sufficient condition is given for these spaces to coincide with $H^{p}\left(P^{\mu}\right)$, for an arbitrary law $P$ on the state space; further, an application of these results to the construction of Levy systems is presented.

Section 3 contains some new results. We consider [7, Lemma 5.30], which establishes a connection between extremal measures and stable subspaces, and give a generalisation. We prove that this theorem holds for a subspace $G$ of $H^{p}$, where $G$ contains the right continuous modifications of $\left(\mathcal{F}_{t}\right)$-martingales of the form $M_{t}=$ $P\left(A \mid \mathcal{G}_{t}\right)$ for all $A \in\left(\mathcal{G}_{\infty}\right)$, and $\mathbf{F}=\left\{\mathcal{F}_{t}, t \in I\right\}$ is a filtration which is caused by itself. Also, we show that a martingale $N_{t}$ is orthogonal to $\operatorname{stable}(G)$ if $N_{t}$ is orthogonal to the martingales $M_{t}=P\left(A \mid \mathcal{G}_{t}\right)$. These results can be applied to extremal regular weak solutions of stochastic differential equations driven with semimartingales.

\section{Preliminaries and notation}

The study of Granger causality has been mostly concerned with time series (see [3]). But many of the systems to which it is natural to apply tests of causality occur in continuous time, so we will consider continuous time processes.

A probabilistic model for a time-dependent system is described by $\left(\Omega, \mathcal{F}, \mathcal{F}_{t}, P\right)$, where $(\Omega, \mathcal{F}, P)$ is a probability space and $\left\{\mathcal{F}_{t}, t \in I\right\}$ is a 'framework' filtration, that is, $\left(\mathcal{F}_{t}\right)$ are all events in the model up to and including time $t$ and constitute a subset of $(\mathcal{F})$. We suppose that the filtration $\left(\mathcal{F}_{t}\right)$ satisfies the 'usual conditions', which means that $\left\{\mathcal{F}_{t}, t \in I\right\}$ is right continuous and each $\left(\mathcal{F}_{t}\right)$ is complete.

An analogous notation will be used for filtrations $\mathbf{H}=\left(\mathcal{H}_{t}\right), \mathbf{G}=\left(\mathcal{G}_{t}\right)$ and $\mathbf{J}=\left(\mathcal{J}_{t}\right)$.

A family of $\sigma$-algebras induced by a stochastic process $X=\left\{X_{t}, t \in I\right\}$ is given by $\mathbf{F}^{X}=\left\{\mathcal{F}_{t}^{X}, t \in I\right\}$, where $\mathcal{F}_{t}^{X}=\sigma\left\{X_{u}, u \in I, u \leq t\right\}$, being the smallest $\sigma$-algebra with respect to which the random variables $X_{u}, u \leq t$, are measurable. The process $X_{t}$ is $\left(\mathcal{F}_{t}\right)$-adapted if $\left(\mathcal{F}_{t}^{X}\right) \subseteq\left(\mathcal{F}_{t}\right)$ for each $t$.

The intuitively plausible notion of causality is given in [2] and generalised in [11] for families of Hilbert spaces. Now, it is natural to introduce the following definition of causality between filtrations.

Definition 2.1 (Compare with $[11,17]$ ). It is said that $\mathbf{G}$ causes $\mathbf{J}$ within $\mathbf{H}$ relative to $P$ (and written as $\mathbf{J}<\mathbf{G} ; \mathbf{H} ; P$ ) if $\mathcal{J}_{\infty} \subseteq \mathcal{H}_{\infty}, \mathbf{G} \subseteq \mathbf{H}$ and if $\left(\mathcal{J}_{\infty}\right)$ is conditionally independent of $\left(\mathcal{H}_{t}\right)$ given $\left(\mathcal{G}_{t}\right)$ for each $t$. If there is no doubt about $P$, we omit 'relative to $P$ '.

The essence of this defintion is that all information about $\left(\mathcal{J}_{\infty}\right)$ that gives $\left(\mathcal{H}_{t}\right)$ comes via $\left(\mathcal{G}_{t}\right)$ for arbitrary $t$; equivalently, $\left(\mathcal{G}_{t}\right)$ contains all information from the $\left(\mathcal{H}_{t}\right)$ needed for predicting $\left(\mathcal{J}_{\infty}\right)$.

If $\mathbf{G}$ and $\mathbf{H}$ are such that $\mathbf{G} K \mathbf{G} ; \mathbf{H}$, we shall say that $\mathbf{G}$ is its own cause within $\mathbf{H}$ (compare with [11]). 
This definition can be applied to stochastic processes: it will be said that stochastic processes are in a certain relationship if and only if the corresponding induced filtrations are in this relationship. For example, an $\left(\mathcal{F}_{t}\right)$-adapted stochastic process $X_{t}$ is its own cause if $\mathbf{F}^{X}=\left(\mathcal{F}_{t}^{X}\right)$ is its own cause within $\mathbf{F}=\left(\mathcal{F}_{t}\right)$, that is, if

$$
\mathbf{F}^{X}<\mathbf{F}^{X} ; \mathbf{F} ; P \text {. }
$$

The process $X$ which is its own cause is completely described by its behaviour relative to $\mathbf{F}^{X}$.

Proposition 2.2 [14]. A Brownian motion $W=\left(W_{t}, t \in I\right)$ on a filtered probability $\operatorname{space}\left(\Omega, \mathcal{F}, \mathcal{F}_{t}, P\right)$ is its own cause within $\mathbf{F}=\left\{\mathcal{F}_{t}, t \in I\right\}$ relative to probability $P$.

The assertion $\mathbf{G}<\mathbf{G} ; \mathbf{H} ; P$ implies that $\mathcal{G}_{t}=\mathcal{H}_{t} \cap \mathcal{G}_{\infty}$ for every $t \geq 0$. Also, $\left(\mathcal{G}_{t}\right)$ is a filtration generated by continuous martingales of the form $P\left(A \mid \mathcal{H}_{t}\right), A \in\left(\mathcal{G}_{\infty}\right)$.

The following definition is concerned with extremal measures.

Definition 2.3 [16]. A probability measure $P$ of $\mathcal{P}$ is called extremal if whenever $P=\alpha Q+(1-\alpha) R$ with $0<\alpha<1, Q, R \in \mathcal{P}$, then $P=Q=R$.

The next theorem shows that the notion of extremal measures is closely connected with the concept of causality.

Proposition 2.4 [9]. Let $\left(\Omega, \mathcal{G}_{\infty}, P\right)$ be a probability space with a filtration $\left(\mathcal{G}_{t}\right)$. Let $G$ be a set of $\left(\mathcal{G}_{t}, P\right)$-martingales. Then the following statements are equivalent.

(i) $P$ is extremal in $\mathcal{P}$, the set of all probability measures $Q$ on $\left(\mathcal{G}_{\infty}\right)$ which coincide with $P$ on $\mathcal{G}_{-\infty}=\bigcap_{t} \mathcal{G}_{t}$, and under which all elements of $G$ are $\left(\mathcal{G}_{t}, Q\right)$ martingales.

(ii) For any filtration $\left(\overline{\mathcal{F}}_{t}\right)$ on an extension $(\bar{\Omega}, \overline{\mathcal{F}}, \bar{P})$ of $\left(\Omega, \mathcal{G}_{\infty}, P\right)$, if $\left(\overline{\mathcal{F}}_{t}\right) \geq\left(\overline{\mathcal{G}}_{t}\right)$ and if all elements of $G$ are $\left(\mathcal{F}_{t}\right)$-martingales then

$$
\overline{\mathbf{G}} \mid<\overline{\mathbf{G}} ; \overline{\mathbf{F}} ; \bar{P} .
$$

The concept of causality is invariant under changes of probability measure, as is shown by the following lemma (see [12]).

Lemma 2.5 [9]. In the measurable space $(\Omega, \mathcal{F})$ let the filtrations $\mathbf{H}=\left\{\mathcal{H}_{t}\right\}, \mathbf{G}=\left\{\mathcal{G}_{t}\right\}$ and $\mathbf{F}=\left\{\mathcal{F}_{t}\right\}$ be given and let $P$ and $Q$ be probability measures on $\mathcal{F}$ satisfying $Q \ll P$ with $d Q / d P$ as $\left(\mathcal{F}_{\infty}\right)$-measurable. Then

$$
\mathbf{G} K \mathbf{H} ; \mathbf{F} ; P \text { implies } \mathbf{G}<\mathbf{H} ; \mathbf{F} ; Q \text {. }
$$

Let $\left(\Omega, \mathcal{F}, \mathcal{F}_{t}, P\right)$ be a filtered probability space with $\left(\mathcal{F}_{t}\right)$ right continuous and complete. Let $\mathcal{M}$ be the space of right continuous, uniformly integrable $\left(\mathcal{F}_{t}, P\right)$ martingales with seminorm $\left\|\left(N_{t}\right)\right\|_{\mathcal{M}}=\left\|N_{\infty}\right\|_{L^{1}}$, and let $H^{p}, p \in[1, \infty)$, be the set of martingales $N_{t} \in \mathcal{M}$ which satisfy $\left\|N_{t}\right\|_{H^{p}}^{p}=E\left(\sup _{t}\left|N_{t}\right|^{p}\right)<\infty$.

Definition 2.6 [7]. A closed linear subspace $\mathcal{X}$ of $H^{p}$ is called a stable subspace if it is stable under stopping, that is, if $X \in \mathcal{X}$ then $X^{T} \in \mathcal{X}$ for every stopping time $T$. If $\mathcal{X}$ 
is a subset of $H^{p}$ then the smallest closed linear subspace of $H^{p}$ which contains $\mathcal{X}$ is denoted by $\operatorname{stable}_{p}(\mathcal{X})$.

Definition 2.7 [15]. Two martingales $M$ and $N$ are said to be weakly orthogonal if $E\left(M_{\infty} N_{\infty}\right)=0$.

There is, however, another, stronger notion of orthogonality for martingales.

Definition 2.8 [15]. Two martingales $M$ and $N$ are said to be strongly orthogonal if their product $M N$ is a martingale.

If $M$ and $N$ are strongly orthogonal martingales they are weakly orthogonal, too. However, the converse is not true. For a set $U$, let $U^{\perp}$ (respectively, $U^{\times}$) denote the set of all elements of $\mathcal{M}^{2}$ orthogonal (respectively, strongly orthogonal) to each element of $U$.

Theorem 2.9 [15]. Let $U$ be a subset of $\mathcal{M}^{2}$ which is stable. Then $U^{\perp}$ is a stable subspace, and every element of $U^{\perp}$ is strongly orthogonal to every element of $U$ (in other words, $U^{\times}=U^{\perp}$ ) and the stable subspace generated by $U$ is $U^{\perp \perp}=U^{\times \perp}=U^{\times \times}$.

\section{Causality and stable subspaces}

In this section we consider stable subspaces of $L^{1}$, due to Kunita and Watanabe, which concern stable subspaces of some topological spaces of martingales associated with a Markov process (see [6]).

Obviously, $H^{p}$ is a stable subspace. The intersection of stable subspaces is also stable, hence $\operatorname{stable}_{p}(G)$ is meaningful for every $G \subseteq H^{p}$. To make the notation as simple as possible, if the subscript $p$ is not important we shall drop it and instead of stable $_{p}(G)$ we shall simply write stable $(G)$. Of course, stable $(G)$ is the smallest stable subspace of $H^{p}$ which contains $G$.

Let $G$ be a set of right continuous modifications of the martingales $P\left(A \mid \mathcal{G}_{t}\right)$ for all $A \in \mathcal{G}_{\infty}$, or

$$
G=\left\{M_{t}=\left(P\left(A \mid \mathcal{G}_{t}\right)\right) \mid A \in \mathcal{G}_{\infty}\right\} .
$$

Theorem 3.1. Suppose that the condition $\mathbf{G}<\mathbf{G} ; \mathbf{F} ; P$ holds. If $N_{t}$ is orthogonal to $G$ for all $N_{t} \in \mathcal{M}$, then $N_{t}$ is orthogonal to $\operatorname{stable}(G)$.

Proof. Let us denote by $\mathcal{Y}$ the set of $\left(\mathcal{F}_{t}\right)$-martingales which are orthogonal to $N_{t}$. Since $G \subseteq \mathcal{Y}$, it is sufficient to prove that $\mathcal{Y}$ is a stable subspace over $\left(\mathcal{F}_{t}\right)$.

As we remarked, $\mathcal{Y}$ is closed under stopping. If $M_{t}=P\left(A \mid \mathcal{G}_{t}\right), A \in\left(\mathcal{G}_{\infty}\right)$ is a right continuous $\left(\mathcal{F}_{t}\right)$-martingale, to prove that $\mathcal{Y}$ is closed under stopping we need to prove that the process $M^{T}=M_{t \wedge T}$ is a right continuous $\left(\mathcal{F}_{t}\right)$-martingale. Clearly, $M^{T}$ is right continuous. Note that if $X$ is adapted and cadlag and if $T$ is a stopping time, then

$$
M_{t}^{T}=M_{T \wedge t}=M_{t} 1_{\{t<T\}}+M_{T} 1_{\{t \geq T\}}
$$


is adapted, too. Then, by Doob's optional sampling theorem [15, Theorem 2.16],

$$
\begin{aligned}
M_{t \wedge T} & =E\left(M_{T} \mid \mathcal{F}_{t \wedge T}\right)=E\left(M_{T} 1_{\{T<t\}}+M_{T} 1_{\{T \geq t\}} \mid \mathcal{F}_{t \wedge T}\right) \\
& =M_{T} 1_{\{T<t\}}+E\left(M_{T} 1_{\{T \geq t\}} \mid \mathcal{F}_{t \wedge T}\right)=M_{T} 1_{\{T<t\}}+E\left(M_{T} \mid \mathcal{F}_{t}\right) 1_{\{T \geq t\}} .
\end{aligned}
$$

Therefore

$$
M_{T \wedge t}=M_{T} 1_{\{T<t\}}+E\left(M_{T} \mid \mathcal{F}_{t}\right) 1_{\{T \geq t\}}=E\left(M_{T} \mid \mathcal{F}_{t}\right),
$$

since $M_{T} 1_{\{T<t\}}$ is $\left(\mathcal{F}_{t}\right)$-measurable. Thus, $M^{T}$ is an $\left(\mathcal{F}_{t}\right)$-martingale by [15, Theorem 2.13].

If $N_{t}$ is a martingale orthogonal to $M_{t}$, we need to prove that $N_{t}$ will be orthogonal to the stopped process $M^{T}=M_{t \wedge T}$. Namely, the process $M^{T} N$ should be a local martingale. Then, according to [1, Theorem 9],

$$
\begin{aligned}
E\left(M_{\infty}^{T} N_{\infty} \mid \mathcal{F}_{t}\right) & =E\left(N_{\infty} M^{T} \mid \mathcal{F}_{t}\right)=E\left(N_{\infty} P\left(A \mid \mathcal{G}_{T}\right) \mid \mathcal{F}_{t}\right) \\
& =E\left(N_{\infty} P\left(A \mid \mathcal{F}_{T}\right) \mid \mathcal{F}_{t}\right)=E\left(N_{\infty} \mid \mathcal{F}_{t}\right) E\left(E\left(1_{A} \mid \mathcal{F}_{T}\right) \mid \mathcal{F}_{t}\right) \\
& =N_{t} E\left(1_{A} \mid \mathcal{F}_{T} \cap \mathcal{F}_{t}\right)=N_{t} \cdot \begin{cases}E\left(1_{A} \mid \mathcal{F}_{t}\right), & t \leq T \\
E\left(1_{A} \mid \mathcal{F}_{T}\right), & T<t\end{cases} \\
& =N_{t} \cdot\left\{\begin{array}{ll}
M_{t}, & t \leq T \\
M_{T}, & T<t
\end{array}=N_{t} M^{T},\right.
\end{aligned}
$$

where we use the relation $\mathbf{G} \mid<\mathbf{G} ; \mathbf{F} ; P$, which means that

$$
\forall A \in\left(\mathcal{G}_{\infty}\right), \quad P\left(A \mid \mathcal{G}_{t}\right)=P\left(A \mid \mathcal{F}_{t}\right) .
$$

So $M^{T} N$ is a martingale, and $M^{T}$ and $N$ are orthogonal martingales.

To prove that $\mathcal{Y}$ is a stable subspace, suppose that $M_{n} \in \mathcal{Y}$ is a sequence of martingales converging to $M_{\infty}$ in $\left(\mathcal{F}_{t}\right)$. Let $N_{t} \in \mathcal{M}$. Then $M^{n} N$ is a martingale for each $n$, so $E\left(\left(M_{n} N\right)(T)\right)=0$ for every stopping time $T$. Let $k<\infty$ be an upper bound of $N$. Then

$$
\begin{aligned}
E\left(\left(M_{\infty} N\right)(T)\right) & =\left|E\left(\left(M_{\infty} N\right)(T)\right)-E\left(\left(M_{n} N\right)(T)\right)\right| \\
& \leq E\left|\left(\left(M_{\infty}-M_{n}\right) N\right)(T)\right| \leq k \cdot E\left(\left|\left(M_{\infty}-M_{n}\right)\right|\right) \\
& \leq k \cdot E\left(\sqrt{\left[M_{\infty}-M_{n}\right](\infty)}\right) \leq k \cdot\left\|M_{\infty}-M_{n}\right\|_{H^{p}} \rightarrow 0 .
\end{aligned}
$$

So $M_{\infty} N$ is a martingale, too. Hence, $\operatorname{stable}(G)=\mathcal{Y}=\left\{N \in \mathcal{F}_{t} ; M \perp N\right\}$ is closed in $H^{p}$.

Suppose that $H$ is of the form

$$
H=\left\{R_{t}=\left(P\left(A \mid \mathcal{H}_{t}\right)\right) \mid A \in \mathcal{H}_{\infty}\right\} .
$$

A necessary and sufficient condition for $\operatorname{stable}(H)=H^{p}$ is that every bounded martingale orthogonal to $H$ be zero.

This condition can be transformed into an extremal property for the measure $P$ (see [8]). Suppose that $\mathcal{P}$ denotes the set of all the probability measures $Q$ which are absolutely continuous with respect to $P(Q \ll P)$ and such that: 
(A) $Q=P$ on $\left(\mathcal{F}_{0}\right)$;

(B) every element of $H$ is a martingale with respect to the filtration $\left(\mathcal{F}_{t}\right)$ and the probability measure $Q$.

Note that the second condition would not be meaningful if $Q$ were not assumed to be absolutely continuous with respect to $P$ : the elements of $H$ are classes of indistinguishable processes and all $P$-negligible sets are added to $\left(\mathcal{F}_{0}\right)$. That is to say, the next result holds.

Theorem 3.2. Let $H$ be a subset of $H^{p}, 1 \leq p<\infty$, such that $1 \in H$. Then stable $(H)=$ $H^{p}$ if and only if $\mathbf{H} K \mathbf{H} ; \mathbf{F} ; P$.

Proof. Let $\mathbf{H} \mid<\mathbf{H} ; \mathbf{F} ; P$ hold. Suppose that there exists a probability measure $Q \in \mathcal{P}$ which is absolutely continuous relative to $P$ and satisfies conditions (A) and (B). Then, by Lemma $2.5, \mathbf{H} \mid<\mathbf{H} ; \mathbf{F} ; Q$ holds. Obviously, $H$ is a set of $\left(\mathcal{F}_{t}, P\right)$-martingales and, using Proposition $2.4\left(\right.$ set $\left.\overline{\mathcal{G}}_{t}=\mathcal{H}_{t}, \overline{\mathcal{F}}_{t}=\mathcal{F}_{t}\right)$ it follows that $P$ is an extremal point in the set $\mathcal{P}$ of probability measures on $\left(\mathcal{H}_{\infty}\right)$. To prove that stable $(H)=H^{p}$ it is necessary and sufficient that every bounded martingale orthogonal to $H$ be zero. If $P$ is an extremal measure, then $P=Q$ where $L_{\infty}=d Q / d P=1$ and $L_{t}=E\left(L_{\infty} \mid \mathcal{F}_{t}\right)=1$. By assumption (A), $L_{0}=1$, and by assumption (B), $L_{t}-L_{0}=1-1=0$ and that martingale is equal to zero, so stable $(H)=H^{p}$.

Conversely, suppose that stable $(H)=H^{p}$; then all bounded martingales orthogonal to $H$ are equal to zero. Suppose $L_{t}=E\left(L_{\infty} \mid \mathcal{F}_{t}\right)$, where $L_{t}-L_{0}=0$, and $L_{t}-L_{0}$ is orthogonal to $H$.

Let

$$
L_{t}=E\left(\frac{d Q}{d P} \mid \mathcal{F}_{t}\right),
$$

where $L_{\infty}=d Q / d P$ is $\left(\mathcal{H}_{\infty}\right)$-measurable and $R_{t}=P\left(A \mid \mathcal{H}_{t}\right) \in H$. Measures $P, Q \in \mathcal{P}$ as well. By assumption, elements of $H$ must be $\left(\mathcal{F}_{t}, P\right)$-martingales. All elements of $H$ are $\left(\mathcal{F}_{t}, Q\right)$-martingales because of condition (B), so

$$
\begin{gathered}
E_{Q}\left(R_{\infty} \mid \mathcal{F}_{t}\right)=R_{t}=E_{P}\left(R_{\infty} \mid \mathcal{F}_{t}\right), \\
E_{Q}\left(P\left(A \mid \mathcal{H}_{\infty}\right) \mid \mathcal{F}_{t}\right)=E_{P}\left(P\left(A \mid \mathcal{H}_{\infty}\right) \mid \mathcal{F}_{t}\right), \\
E_{Q}\left(\chi_{A} \mid \mathcal{F}_{t}\right)=E_{P}\left(\chi_{A} \mid \mathcal{F}_{t}\right), \\
Q\left(A \mid \mathcal{F}_{t}\right)=P\left(A \mid \mathcal{F}_{t}\right),
\end{gathered}
$$

where the indicator function $\chi_{A}$ is $\left(\mathcal{H}_{\infty}\right)$-measurable. So $P$ is an extremal point of the set of all measures $\mathcal{P}$. By Proposition 2.4,

$$
\mathbf{H} K \mathbf{H} ; \mathbf{F} ; P \text {. }
$$

This concludes the proof.

Corollary 3.3. Suppose that sets $G$ and $H$ are subsets of $H^{p}$. Then $\mathbf{G} K \mathbf{G} ; \mathbf{F} ; P$ and $\mathbf{H} \mid<\mathbf{H} ; \mathbf{F} ; P$ if and only if $\operatorname{stable}(G)=\operatorname{stable}(H)$. 
Proof. The proof follows immediately from Theorem 3.2. From the conditions $\mathbf{G} \mid<\mathbf{G} ; \mathbf{F} ; P$ and $\mathbf{H}<\mathbf{H} ; \mathbf{F} ; P$, it follows that $\operatorname{stable}(G)=H^{p}$ and stable $(H)=H^{p}$, so $\operatorname{stable}(G)=H^{p}=\operatorname{stable}(H)$.

Theorem 3.1 yields that

$$
\forall N_{t} \in \mathcal{M}, \quad N_{t} \perp G \Rightarrow N_{t} \perp \operatorname{stable}(G) .
$$

The reason why (3.2) is interesting is shown in [5]. It relates the concept of stable subspaces of $H^{p}$ for different values of $p$. Using the duality between $H^{p}$ and $H^{q}$ $(1 / p+1 / q=1, p \neq 1)$ together with the Hahn-Banach theorem, (3.2) implies that, for all $p \in[1, \infty)$,

$$
\operatorname{stable}_{p}(G)=\operatorname{stable}_{1}(G) \cap H^{p} .
$$

Condition (3.2) connects extremality of measure with the concept of causality. Suppose that $\mathcal{F}_{0}$ is a $0-1 \sigma$-field; then $P$ is an extremal measure in the set $\mathcal{P}$ which denotes the set of measures under which the elements of $G$ are local martingales if and only if $H^{1}=\operatorname{stable}_{1}(G \cup\{1\})$ (see [5, Theorem 11.2]). If $Q$ is a measure from $\mathcal{P}$ which is absolutely continuous relative to $P$, then the orthogonality condition (3.2) (where we set $N_{\infty}=d Q / d P$ ) is equivalent to

$$
Q \in \mathcal{P}, \quad Q \ll P \Rightarrow Q=P .
$$

In other words, elements of stable $_{1}(G)$ remain martingales under any measure $Q \ll P$, so, for all $A \in\left(\mathcal{G}_{\infty}\right), Q\left(A \mid \mathcal{F}_{t}\right)=P\left(A \mid \mathcal{F}_{t}\right)$. By [5, Theorem 12.21], (3.2) can be applied to solutions of martingale problems.

Remark 3.4. If $\mathcal{P}$ consists of a single element $P$, this probability measure is extremal. This triviality is fundamental in applications.

Corollary 3.3 can be applied to solutions of martingale problems and to extremal regular weak solutions of stochastic differential equations. For example, let us consider the equation

$$
\left\{\begin{array}{l}
d X_{t}=u_{t}(X) d Z_{t} \\
X_{0}=0
\end{array}\right.
$$

where $Z_{t}$ is an $m$-dimensional semimartingale, and $u_{t}(X)$ is an $(n \times m)$-dimensional predictable functional. Suppose that the set of objects $\left(\Omega, \mathcal{F}, \mathcal{F}, P, X_{t}, Z_{t}\right)$ is a regular weak solution of (3.3) (see [14]).

Let us assume that $\left(\mathcal{F}_{0}\right)$ is complete, $H$ is the set of right continuous modifications of the martingales $L_{t}=P\left(A \mid \mathcal{F}_{t}^{X, Z}\right)$ for $A \in\left(\mathcal{F}_{\infty}^{X, Z}\right)$ and $\left(\mathcal{G}_{t}\right)=\left(\mathcal{F}_{t}^{Z}\right)$ in (3.1). From the definition of weak solution we have that $\mathbf{F}^{Z} \mid<\mathbf{F}^{Z} ; \mathbf{F} ; P$ holds (see [14]). According to [14, Theorems 4.4 and 4.3], an extremal weak solution of equation (3.3) satisfies the condition $\mathbf{F}^{X, Z}<\mathbf{F}^{X, Z} ; \mathbf{F} ; P$. So, by Corollary 3.3, for an extremal regular weak solution of the equation (3.3) we have, for any $p>1$,

$$
\operatorname{stable}_{p}(G)=\operatorname{stable}_{p}(H),
$$


where $\operatorname{stable}_{p}(H)$ is the smallest stable subspace over $\left(\mathcal{F}_{t}\right)$ and $\operatorname{stable}_{p}(G)$ is the smallest stable subspace over $\left(\mathcal{F}_{t}^{X, Z}\right)$. An interesting consequence of this is that

$$
\forall A \in\left(\mathcal{F}_{\infty}^{X, Z}\right), \forall t>0, \quad P\left(A \mid \mathcal{F}_{t}^{X, Z}\right)=P\left(A \mid \mathcal{F}_{t}\right),
$$

which links extremality with Granger causality and with the concept of weak uniqueness of the weak solution of the stochastic differential equation of the form (3.3) (see $[10,14])$.

ExAmple 3.5. A trivial example of orthogonal pairs of stable subspaces is: if $T$ is a stopping time, the decomposition

$$
X=X^{T}+\left(X-X^{T}\right)
$$

is an orthogonal decomposition, corresponding to the stable subspace of all martingales stopped at $T$ and that of martingales which are zero on $[0, T]$.

ExAmple 3.6. Let $T>0$ denote arbitrary stopping time. It can be shown that a process of the form $X_{t}^{A}=A 1_{\{t \geq T\}}$ is a uniformly integrable martingale if and only if $A \in L_{1}\left(\mathcal{F}_{T}\right)$ and $E\left(A \mid \mathcal{F}_{T_{-}}\right)=0$. In particular, as $A$ runs through $L_{2}\left(\mathcal{F}_{T}\right) \oplus L_{2}\left(\mathcal{F}_{T-}\right), X^{A}$ runs through the stable subspace of martingales which are stopped at $T$ and are zero on $[0, T)$. An immediate calculation shows that it consists of the martingales $Y$ such that $Y_{T}$ is $\left(\mathcal{F}_{T-}\right)$-measurable. The corresponding orthogonal decomposition is $Y=Z+W$, where

$$
Z_{t}=\left(Y_{T}-E\left(Y_{T} \mid \mathcal{F}_{T-}\right)\right) 1_{\{t \geq T\}}, \quad W_{t}=Y_{t}-Z_{t}
$$

\section{References}

[1] P. Bremaud and M. Yor, 'Changes of filtrations and of probability measures', Z. Wahrscheinlichkeitstheorie verw. Gebiete 45 (1978), 269-295.

[2] J. B. Gill and Lj. Petrović, 'Causality and stochastic dynamic systems', SIAM J. Appl. Math. 47(6) (1987), 1361-1366.

[3] C. W. J. Granger, 'Investigating causal relations by econometric models and cross spectral methods', Econometrica 37 (1969), 424-438.

[4] V. Grecea, 'On generating $H^{p}$ spaces of martingales', Rev. Roumaine Math. Pures Appl. 53(4) (2008), 317-322.

[5] J. Jacod, Calcul Stochastique et Problèmes de Martingales, Lecture Notes in Mathematics (Springer, Berlin, 1979).

[6] H. Kunita and S. Watanabe, 'On square integrable martingales', Nagoya Math. J. 30 (1967), 209-245.

[7] P. Medvegyev, Stochastic Integration Theory (Oxford University Press, Oxford, 2007).

[8] P. A. Meyer, Probability and Potentials (North-Holland, Amsterdam, 1982).

[9] P. A. Mykland, 'Statistical causality'. Statistical Report No. 14, University of Bergen, 1986.

[10] P. A. Mykland, 'Stable subspaces over regular solutions of martingale problems'. Statistical Report No 15, University of Bergen, 1986.

[11] Lj. Petrović, 'Causality and Markovian representations', Statist. Probab. Lett. 29 (1996), 223-227.

[12] Lj. Petrović and S. Dimitrijević, 'Invariance of statistical causality under convergence', Statist. Probab. Lett. 81 (2011), 1445-1448. 
[13] Lj. Petrović and D. Stanojević, 'Some models of causality and weak solutions of stochastic differential equations with driving semimartingales', Facta Univ. ser. 20 (2005), 103-112.

[14] Lj. Petrović and D. Stanojević, 'Statistical causality, extremal measures and weak solutions of stochastical differential equations with driving semimartingales', J. Math. Model. Algor. 9 (2010), 113-128.

[15] P. Protter, Stochastic Integration and Differential Equations (Springer, Berlin, 2004).

[16] D. Revuz and M. Yor, Continuous Martingales and Brownian Motion (Springer, Berlin, 2005).

[17] D. Valjarević and Lj. Petrović, 'Statistical causality and orthogonality of local martingales', Statist. Probab. Lett. 82 (2012), 1326-1330.

LJILJANA PETROVIĆ, Department of Mathematics and Statistics, Faculty of Economics, University of Belgrade, Kamenička 6,

11000 Beograd, Serbia

e-mail: petrovl@ekof.bg.ac.rs

DRAGANA VALJAREVIĆ, Department of Mathematics, Faculty of Science, University of Priština-Kosovska Mitrovica, Lole Ribara 29, 38220 Kosovska Mitrovica, Serbia e-mail: dragana_stan@yahoo.com 